\title{
The influence of female viability differences on the evolution of mate choice
}

\author{
I. P. M. TOMLINSON* \& P. O'DONALD \\ Department of Genetics, University of Cambridge, Downing Street, Cambridge CB2 3EH, U.K.
}

\begin{abstract}
When females choose a mate, they may do so on the basis of some arbitrary character or they may prefer a trait that provides them with some direct or indirect benefit. One class of models of female choice, collectively referred to as the 'handicap principle', states that preferred ornaments act as markers of underlying heritable male fitness. 'Handicap' models have proved to be of great importance in explaining how female mating preferences can coevolve with male ornaments by an augmented form of the 'Fisherian process'. We suggest that differences in heritable female (Darwinian) fitness might affect the evolution of female mating preferences. Although little experimental evidence currently exists, it is entirely plausible that female fitness affects the expression of mating preferences. For example, fitter females might undertake more rigorous searches for rare, preferred males. In order to determine whether differences in female fitness can influence sexual selection by female choice, a model is presented in which mating preferences are more likely to be expressed by females of higher fitness. Results of the model show how specific female preferences for arbitrary, disadvantageous male characters can easily evolve, even if choice itself is costly. In many of these cases, the Fisherian process alone would not be sufficient to cause coevolution of preference and preferred character. Polymorphisms in male characters may also be maintained in the fitter female models. Some of the models show extreme and unusual fluctuations in the frequencies of male characters and female preferences before equilibrium is reached. Differences in female fitness may have important influences on sexual selection by female choice.
\end{abstract}

Keywords: female choice, female viability, sexual selection.

\section{Introduction}

Models of sexual selection by female choice vary considerably in their complexity and mathematical sophistication. One of the most contentious aspects of models based on the 'handicap principle' (Zahavi, 1975; West-Eberhard, 1979; Hamilton \& Zuk, 1982) has been the existence of additive genetic variation in male fitness. It is now generally accepted that such variation can exist and can influence the expression of male ornaments. If so, it follows that heritable variation can also exist in female fitness and affect the expression of female mating preferences.

We suggest that differences in female Darwinian fitness may have important influences on female choice. Although little experimental evidence

${ }^{*}$ Correspondence: Cancer Genetics Laboratory, Imperial Cancer Research Fund, 44 Lincoln's Inn Fields, London WC2A 3PX, U.K. currently exists, female fitness may correlate with the expression of a mating preference for several reasons: (i) fitter females may emerge or be born earlier in the breeding season and have the first pick of mates, or more time to assess potential mates; (ii) it may pay fitter females to assess a number of potential mates, whereas less fit females, as a strategy, might mate with less choosiness; fitter females may, for example, be able to tolerate a number of rejections of males whereas unfit, choosy females may, in extremis, die before mating; (iii) females may 'compete' in some way for the pick of the males, at a lek, for example; and (iv) fitter females may encounter more males and therefore be less likely to miss preferred males.

In order to examine the possibility that fitter females are more likely to express a preference, mathematical models of sexual selection by female choice have been set up. Simulations have been used to determine (i) whether preference alleles can correlate with increased Darwinian fitness (as a 
result of the rules governing preference expression) and thereby boost coevolution of the preference and preferred character and (ii) whether differences in female fitness can explain how preference alleles can evolve from a low initial frequency to those reported (e.g. a frequency of greater than 0.3 in the two-spot ladybird, Adalia bipunctata; O'Donald \& Majerus, 1992). The models incorporate Fisher's $(1930,1958)$ process of coevolution of male characters and their female preferences. Costs of choice are also assumed in some cases. The 'fitter female' models are comparable with those handicap models that can 'reinforce' or 'facilitate' the Fisherian process (Pomiankowski, 1987a; Tomlinson, 1988a), owing to genetic correlation of the male character with higher heritable fitness. The equivalent correlation in the handicap models is between female preference and higher fitness. It will be determined whether this correlation develops and causes the handicap models to have a similar effect on the Fisherian process to the handicap models.

\section{Models and results}

In the three models presented (see Appendix), the population is diploid, mates polygynously and is infinite. Dominant alleles at two unlinked loci determine a selectively disadvantageous preferred trait $(A)$ and a female preference $(B)$. The models are therefore based on O'Donald's diploid model of sexual selection by female choice (see O'Donald, 1980 for details). In this study, a dominant allele $(C)$ at a third, unlinked locus confers extra fitness. Selective coefficients are additive. As in the two-spot ladybird, neither the preferred trait nor extra fitness is considered to be sex-limited. The expression of the preferred character is assumed not to be influenced by an individual's genotype at the ' $C$ ' locus. The preferred character is generally assumed to be present at a low frequency initially, in order to promote its coevolution with the preference (O'Donald, 1980). In general, the assumptions regarding genetic control of all three traits and additive selective coefficients do not affect the qualitative behaviour of the models.

Computer simulations of evolution have been run with various values of the following variables: extra fitness conferred by allele $C$; costs of preference; disadvantage associated with the preferred male character; and initial frequency of the preference allele $B$. Additive genetic variance in fitness is maintained. Consequently, a new fitness allele is introduced into the population whenever the frequency of the previous fitness allele rises over 0.995. The simulations are continued until the population reaches some point of interior or boundary equilibrium.

\section{Complete, epistatic expression of preference: the 'CE' model}

This model is intended to simulate a situation favourable to the coevolution of the preference and preferred character. Here, the only females that express a preference are those which possess the alleles for both the preference and for extra fitness. There is therefore epistasis between the preference and fitness genes. The expression of preference is 'complete', that is all females with a preference allele mate with a preferred male, irrespective of male frequency. There is no cost of female preference.

The simulations show that the incorporation of differences in female fitness can indeed cause a female preference allele to rise from a low initial frequency to observed levels. There is a relatively broad (and arguably realistic) band of initial conditions and fitnesses that allows the preferred character to evolve and which promotes higher preference gene frequencies. Examples are shown in Table 1 and Fig. 1. In other cases, the preference and preferred character are lost from the population. It

Table 1 The CE model. The preference, character and extra fitness alleles are all present at initial frequencies of 0.001 and a further fitness allele $C$ is reintroduced at this frequency when its frequency has risen above 0.995 . The last two lines in the table show for comparison a situation in which preference expression is independent of female fitness (that is, with the Fisherian process alone operating)

\begin{tabular}{lccc}
\hline$S_{\text {a }}$ & $S_{\mathrm{c}}$ & $A_{\text {eq }}$ & $B_{\text {eq }}$ \\
\hline 0.05 & 0.30 & 1.0 & 0.04 \\
0.20 & 0.30 & 1.0 & 0.19 \\
0.40 & 0.30 & 1.0 & 0.48 \\
0.60 & 0.30 & 1.0 & 0.84 \\
0.20 & 0.05 & 1.0 & 0.22 \\
0.20 & 0.30 & 1.0 & 0.19 \\
0.20 & 0.40 & 1.0 & 0.18 \\
0.20 & 0.70 & 1.0 & 0.15 \\
0.05 & 0.00 & 0.0 & 0.00 \\
0.20 & 0.00 & 0.0 & 0.00 \\
\hline
\end{tabular}

$S_{\mathrm{a}}$ is the selective disadvantage to the preferred character (allele $A$ ); $S_{\mathrm{c}}$ is the advantage to the extra fitness allele $C$. $A_{\text {eq }}$ and $B_{\text {eq }}$ are the equilibrium frequencies of the character and preference alleles, respectively. 


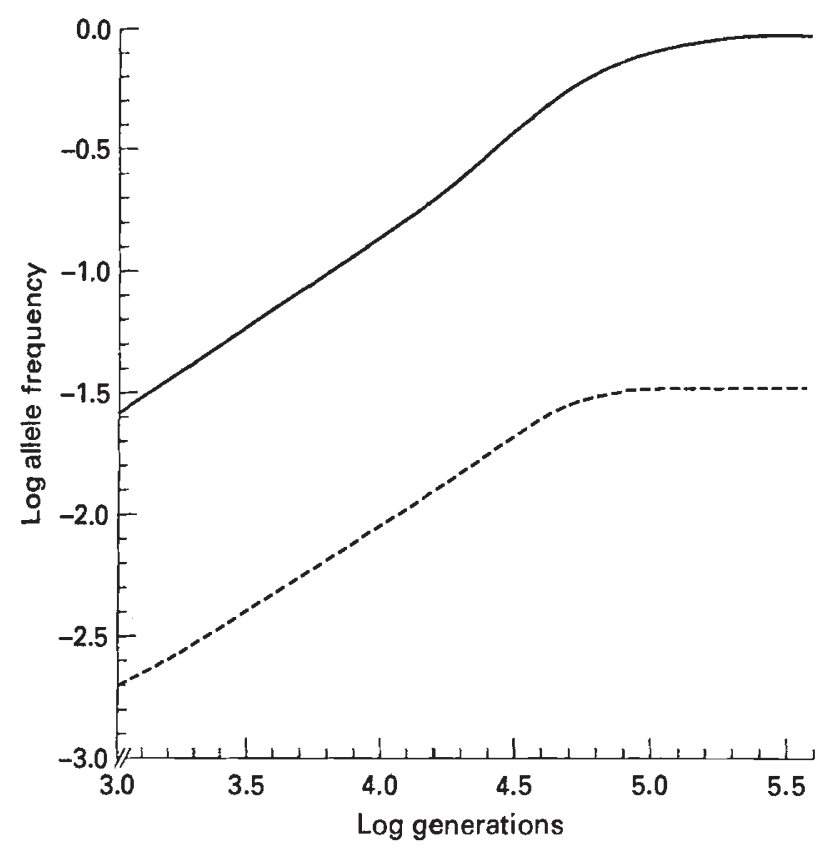

Fig. 1 Coevolution of alleles $A$ (solid line) and $B$ (dashed line) under the $C E$ model. Initially, alleles $A, B$ and $C$ are present at a frequency of 0.001 . $C$ is reintroduced into the population every time its frequency rises above $0.995 . A$ carries an natural selective disadvantage of 0.2 and $C$ carries an advantage of 0.3. Evolution is slow here, but in the equivalent model without fitness differences, the preference and preferred character are lost from the population. The graph is confined to evolution from 1000 generations onwards and the initial decline in the frequency of the preferred character and preference alleles is therefore not shown.

is not possible to find an analytical solution to the model to define the range of initial values which cause the frequency of preference and character to rise.

The coevolutionary process takes place in two stages under the CE model (Figs 1 and 2). When a new fitness allele first arises in the population, the number of preferring females is small, as few possess both the preference and the fitness alleles. Consequently, the preference is insufficiently strong to support the disadvantageous character in the population. The preferred character and preference decline in frequency (not shown in Fig. 1), although the fitness allele spreads through the population as expected. At this stage, the preference and extra fitness are actually negatively correlated (Fig. 2), because females with both these genes are being selected out of the population.

The second phase of the coevolutionary process then occurs (Figs 1 and 2). By now, the frequency of

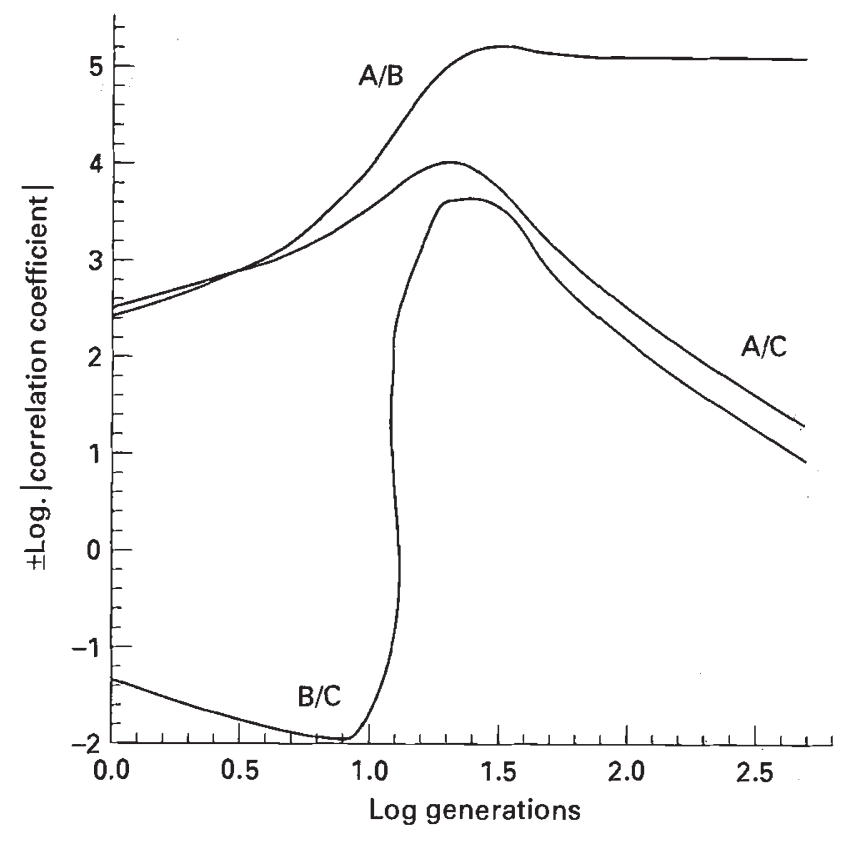

Fig. 2 The correlation coefficients under the $\mathrm{CE}$ model between alleles $A, B$ and $C$, over the cycle of spread of a single $C$ allele. The correlation coefficient ordinate has been plotted on a logarithmic scale, whilst preserving its sign. Note the change in sign of the correlation between $B$ and $C$. The model uses the same parameters as in Fig. 1.

the preference allele has fallen slightly, whereas the frequency of the fitness gene has risen $\approx 1000$-fold. Therefore, sufficient females can now express a preference that the natural selection which acts against the preferred character can be overcome by sexual selection. The decline in frequency of the preferred character has also helped to give the remaining preferred males a larger, frequency-dependent mating advantage. Positive correlations between the fitness and preference genes build up and the preference and preferred character spread through the population to frequencies above those at which they started.

This cycle of slump and recovery is repeated each time a new fitness allele is introduced. Eventually, the preferred character can spread to fixation, the preference having been 'pulled' with the character and fitness genes to frequencies as high as those observed, but generally short of fixation. Table 1 illustrates the effect of changing the fitness parameters on the final character and preference gene frequencies. No internal equilibrium of the preferred character allele is found in this model: the character either spreads to fixation or is lost from the population. The final preference frequency lies between 0 and 1 . 
Table 1 also shows that a larger selective advantage to the extra fitness allele often corresponds to a lower final preference frequency (unless the extra fitness is very low in which case the final preference may also be lower): it is as if an allele conferring much higher fitness spreads through the population too quickly for large correlations to develop between it and the preference. This effect also occurs when successive fitness genes are introduced into the population at an initial frequency of 0.01 rather than 0.001. Again, the fitness allele spreads faster through the population and is correlated less with the mating preference which is not 'pulled' so far through the population.

Perhaps counterintuitively, a more disadvantageous preferred character can also increase final preference gene frequency (although a very high disadvantage retards the coevolutionary process). This result stems from the complete expression of preference assumed in the model. If preferred males are rare, they may gain so many mates from preferential matings that carrying a more disadvantageous character affects their numbers of offspring very little: their frequency prior to mating is lower, but each surviving preferred male simply mates many more times to satisfy all the females with the preference. The correlation between the preference and character genes therefore rises. In consequence, if preferred males do spread through the population, preference may follow to a higher frequency. If the preferred male character is highly deleterious, however, it reduces the number of matings that preferred males have with nonchoosy females. This outweighs the effects of sexual selection and preference and character are lost from the population.

\section{Encounter-dependent expression of preference: the 'ED' model}

In this model, expression of preference is 'incomplete' and depends on females randomly encountering a number of potential mates before mating (O'Donald, 1980). A choosy female (that is, with preference allele $B$ ) mates with the first preferred male that she encounters; after a few encounters without meeting a preferred male, however, she gives up and mates at random. Nonchoosy females always mate at random.

It is assumed that a female with allele $B$ and fitness allele $C$ can tolerate $n_{1}$ encounters before mating at random, greater than the corresponding number, $n_{2}$, for a less fit, choosy female (with the genotype $B c$ ). The chances of a $B C$ female mating with a preferred male are therefore greater than those of a $B c$ female, especially when such males are rare. In this way, a correlation may develop between the preference, character and fitness alleles. The ED and CE models converge when $n_{1}$ is very large and $n_{2}$ very small (Table $2 \mathrm{c}$ ).

Results show that the ED model is much less likely to promote the coevolution of a preference and character than the CE model. Very often, the preferred character and preference never undergo any recovery after their initial slump in frequency. The band of initial conditions and fitnesses that allows the preferred character to evolve and promotes higher preference gene frequencies is much narrower in the ED than the $\mathrm{CE}$ model. For example, the conditions which lead to coevolution of preference and character under the CE model (Table 1) rarely do so under the ED model unless $n_{1}$ is very large and $n_{2}$ is very small. Lower preference and character gene frequencies are found at equi-

Table 2 The ED model. Alleles $A, B$ and $C$ are all present at initial frequencies of 0.001 and a further fitness allele $C$ is reintroduced at this frequency when its frequency has risen above 0.995. (a), (b) and (c) are referred to in the text. In (d), preference expression is independent of female fitness (that is, with the Fisherian process alone operating)

\begin{tabular}{lrrrrr}
\hline$S_{\text {a }}$ & $S_{\mathrm{c}}$ & $n_{1}$ & $n_{2}$ & $A_{\text {eq }}$ & $B_{\text {eq }}$ \\
\hline (a) & & & & & \\
0.01 & 0.50 & 50 & 10 & 1.0 & 0.007 \\
0.05 & 0.50 & 50 & 10 & 1.0 & 0.035 \\
0.20 & 0.50 & 50 & 10 & 0.0 & 0.001 \\
(b) & & & & & \\
0.05 & 0.10 & 50 & 10 & 1.0 & 0.044 \\
0.05 & 0.30 & 50 & 10 & 1.0 & 0.040 \\
0.05 & 0.50 & 50 & 10 & 1.0 & 0.035 \\
(c) & & & & & \\
0.20 & 0.30 & 1000 & 2 & 1.0 & 0.170 \\
0.20 & 0.30 & 100 & 2 & 0.0 & 0.000 \\
0.20 & 0.30 & 50 & 2 & 0.0 & 0.000 \\
0.20 & 0.30 & 10 & 2 & 0.0 & 0.000 \\
(d) & & & & & \\
0.05 & 0.00 & 50 & 10 & 0.0 & 0.000 \\
0.20 & 0.00 & 1000 & 2 & 0.0 & 0.000 \\
0.20 & 0.00 & 100 & 2 & 0.0 & 0.000 \\
0.20 & 0.00 & 50 & 2 & 0.0 & 0.000 \\
0.20 & 0.00 & 10 & 2 & 0.0 & 0.000 \\
\hline
\end{tabular}

$S_{\mathrm{a}}, S_{\mathrm{c}}, A_{\text {eq }}$ and $B_{\text {eq }}$ are as defined in Table 1.

$n_{1}=$ number of encounters tolerated by a $B C$ female before she mates at random.

$n_{2}=$ number of encounters tolerated by a $B c$ female before she mates at random. 
librium under the ED model than under the $\mathrm{CE}$ model. These differences arise from the failure of some choosy females to mate preferentially when preferred males are rare in the ED model. This results in lower correlations between the character, preference and fitness alleles. As in the CE model, no internal equilibrium of the preferred character appears to exist in the ED model: the character allele is either lost or fixed. Preference may, however, evolve to some frequency between 0 and 1 . Large $n_{1}$ and small $n_{2}$ generally promote the coevolution of preference and character, as expected by comparison with the CE model (see Table 2c).

When the selective advantage of the extra fitness allele is varied, results are similar to the CE model: lower extra fitness usually gives higher preference frequencies (Table 2b). Similarly, increasing the disadvantage to the preferred character can lead to higher final preference frequencies, although the final preference frequency in the ED model is lower than in the $\mathrm{CE}$ model. In the $\mathrm{ED}$ model, the preferred character need not be severely deleterious for its disadvantage in natural selection to outweigh its mating advantage (as it must be under the CE model). Again, this results from the smaller correlations that develop in the ED model between the character, preference and fitness alleles, especially when the character allele is rare (Table 2a).

When the ED model leads to evolution of the preferred character, the preference allele may not spread to a frequency as high as those observed (see Table 2). There is often a delicate balance: if the preferred character is relatively fit, it may spread quickly to fixation and fail to 'drag' the preference far with it; if it is relatively unfit, it may be lost from the population completely, together with the preference (Table 2c). Nevertheless, even under the ED model, differences in female fitness can give a significant boost to the Fisherian process, because of the correlation of the preference allele with the allele for extra fitness. Preference frequencies similar to those found in $A$. bipunctata can evolve.

\section{Fitter female models and the costs of choice in sexual selection: 'CC' models}

Not all types of 'female choice' will carry costs in natural selection: indeed, some matings might even be less expensive for choosy females. In some cases, however, female choice may carry costs like increased exposure to predators, energy used in searching for mates and the possibility of remaining unmated. Pomiankowski and co-workers (Pomiankowski, 1987b; Pomiankowski et al., 1991) have investigated thoroughly the influence of costly female choice on handicap and Fisherian models of sexual selection by female choice. In general, costly choice prevents the basic Fisherian process from occurring, except transiently. Under circumstances which aid the Fisherian process - for example, some handicap models or with mutation bias acting against male ornaments - the costs of choice can be overcome.

Fitter female models might also have an important effect in overcoming the problems of costly female choice. We have therefore analysed the CE model, in which preference is only expressed by females with both the preference and the fitness alleles, under conditions where choice carries costs. This model has been chosen in order to maximize the effects of the differences in female fitness. It is additionally necessary, of course, to assume that no mutations arise that decouple choice from its dependence on extra fitness. The cost of choice is assumed to be proportional to the frequency of preferring females and to the reciprocal of the frequency of preferred males.

Simulating the $\mathrm{CC}$ variant of the $\mathrm{CE}$ model shows that internal equilibria occur frequently, in contrast to the CE and ED models. Otherwise, the character and preference alleles are lost from the population. The simulations have found no case in which either becomes fixed. There is a unique, stable equilibrium point for each set of fitness parameters, independent of initial gene frequencies. This is to be expected with direct selection on the preference, as it removes a degree of freedom from the Fisherian coevolutionary process. Internal equilibria can occur at values at which there is still considerable cost to preference.

The path of the population to the equilibrium point is often complex, as it depends on many selective factors and genetic interactions, namely: (i) the cost to preference; (ii) the selective disadvantage of the preferred character; (iii) the sexual selective advantage to the preferred character; (iv) extra fitness, associated with the expression of preference; and (v) correlations between genes at all three loci. Figure 3 shows a typical trajectory of the character and preference allele frequencies from their initial values to an internal equilibrium. It seems that initially the preference gives an advantage to the character which consequently spreads through the population and tends to pull the preference with it. The costs of choice mean, however, that the preference does not spread far. When the preferred character is close to fixation, its frequency-dependent mating advantage is lower and the sexual select- 


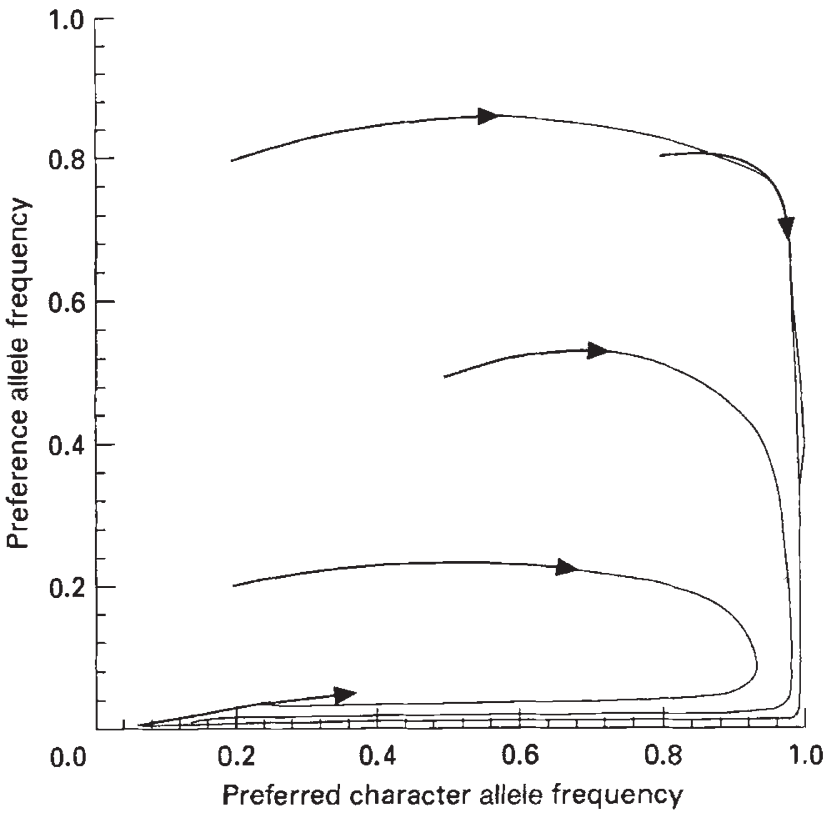

Fig. 3 The approach to equilibrium of the $\mathrm{CC}$ model from several starting frequencies. Allele $C$ is reintroduced into the population at a frequency of 0.01 every time it rises above 0.995 . The selective disadvantage of the preferred character $\left(S_{\mathrm{a}}\right)$ is 0.05 and the extra fitness conferred by allele $C\left(S_{c}\right)$ is 0.2 . The cost of preference is $(0.05 \times$ frequency of choosy females $) /($ frequency of preferred males). Equilibrium is reached at a character allele frequency of about 0.38 and a preference allele frequency of about 0.05 .

ive force pulling the preference frequency upwards is weaker. The cost to preference has diminished little, however, and therefore preference frequency falls. Character frequency does not fall, however, because there remains a large enough number of choosy females to give the character a mating advantage. However, when preference has declined to a low frequency, the mating advantage to the preferred character falls below that needed to overcome its selective disadvantage. The frequency of the preferred character then falls, but the preference is not always lost from the population owing to its remaining association with the extra fitness allele. When the preference has become very rare, cost to preference is low. Preferred males also gain a large mating advantage because they are rare. These factors enable the preference and character to increase in frequency once more. Cost to preference increases, however, because the frequency of the character rises faster than that of the preference. An equilibrium is reached at which the costs of preference and the preferred character balance the mating

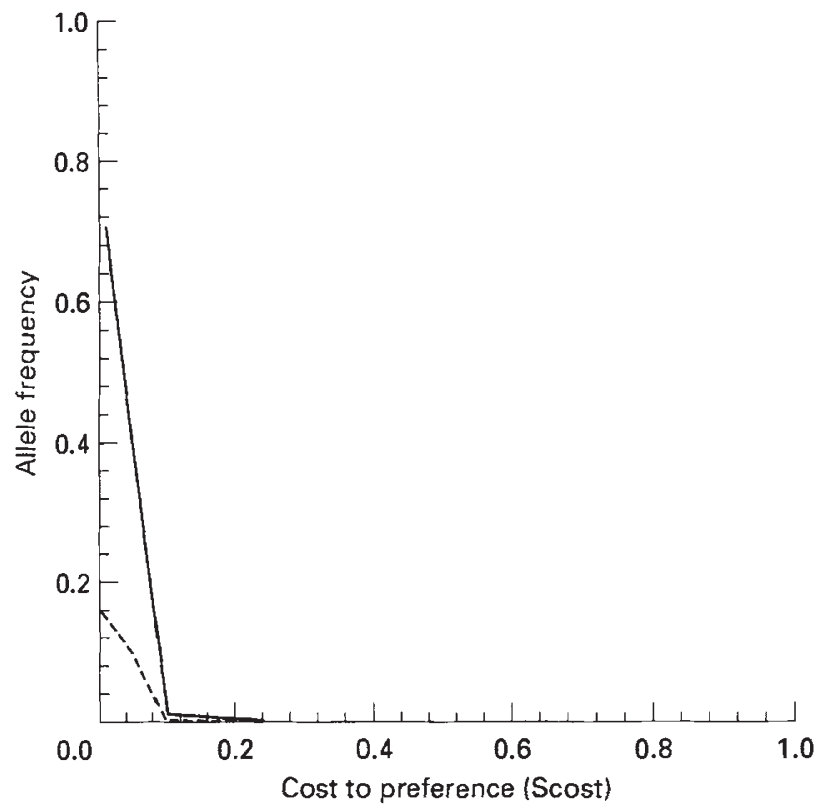

Fig. 4 The dependence of equilibrium allele frequencies (allele $A$, solid line; allele $B$, dashed line) under the $\mathrm{CC}$ model on the cost of preference parameter $\left(S_{\text {cost }}\right) . S_{\mathrm{a}}$ is 0.1 and $S_{\mathrm{c}}$ is 0.2 . Cost of preference is $\left(S_{\text {cost }} \times\right.$ frequency of choosy females $) /($ frequency of preferred males $)$.

advantage to the character and the association of the character and preference with extra fitness. Figures 4, 5 and 6 show the dependence of the preference and character allele frequencies at equilibrium on: (i) the cost of choice; (ii) the disadvantage to the preferred character; and (iii) the advantage conferred by the extra fitness gene.

The cost of choice (Fig. 4) must be small if the preference and character are to be maintained in the population. The selective disadvantage of the preferred character (Fig. 5) must also lie below some threshold value if the preference and character are not to be lost from the population. Up to that threshold, however, final preference gene frequencies are larger as the character becomes increasingly disadvantageous. As in the CE and ED models, this appears to be a result of the increasing influence of the mating preference in determining the spread of the character.

The dependence of equilibrium gene frequencies on the advantage to the extra fitness gene appears to be similar to those in the $\mathrm{CE}$ and $\mathrm{ED}$ models (Fig. 6). At low advantages, the preference and character are lost. If they spread, however, higher equilibrium frequencies are seen when there is a smaller advantage to the extra fitness allele. 


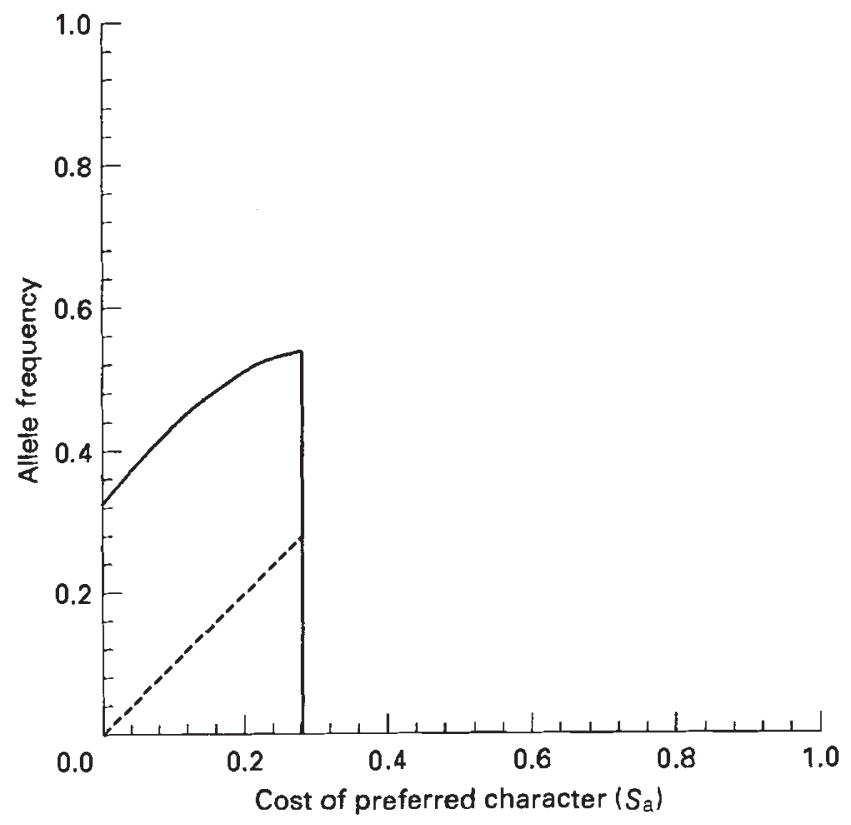

Fig. 5 The dependence of equilibrium allele frequencies (allele $A$, solid line; allele $B$, dashed line) under the CC model on the disadvantage to the preferred character $\left(S_{\mathrm{a}}\right) . S_{\mathrm{c}}$ is 0.2 . Cost of preference is $(0.05 \times$ frequency of choosy females)/(frequency of preferred males). Once $S_{\mathrm{a}}$ has exceeded a threshold, here about 0.285 , preference and character are lost from the population.

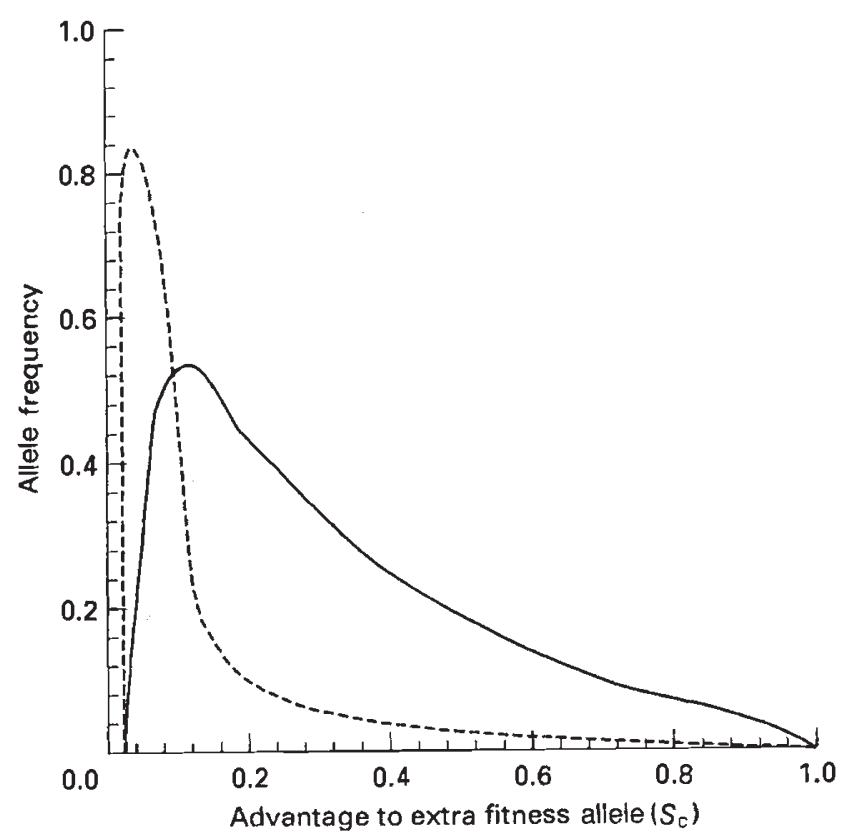

Fig. 6 The dependence of equilibrium allele frequencies (allele $A$, solid line; allele $B$, dashed line) under the $\mathrm{CC}$ model on the advantage given by the extra fitness allele $\left(S_{\mathrm{c}}\right) . S_{\mathrm{a}}$ is 0.1 . Cost of preference is $(0.05 \times$ frequency of choosy female)/(frequency of preferred males).

\section{Conclusions}

The hypothesis that the expression of male ornaments may depend on Darwinian fitness (Zahavi, 1975 ) is one of the most important in the theory of sexual selection. Experimental evidence has not consistently supported this hypothesis (for example, Read, 1987; Read \& Harvey, 1989; John, 1995; Yezerinac \& Weatherhead, 1995), but theoretical analyses have shown that differences in male fitness can have important effects on the coevolution of male characters and female mating preferences (Pomiankowski, 1987a; Tomlinson, 1988a). We have proposed that differences in female Darwinian fitness can influence the expression of mating preferences (in a way analogous to the influence of male fitness on ornaments). Although very few studies of female fitness and mate choice have been undertaken and experimental evidence is lacking, the models presented here show that higher fitness alleles can correlate with alleles controlling male ornaments and female mating preferences, promoting their coevolution. Models in which choice itself carries no costs show that preferred character alleles can become fixed in the population, with preference 'stranded' at some frequency short of fixation, but that internal points of equilibria do not occur. In models in which expression of preference depends on the encounters of females with particular numbers of males, coevolution of preference and character is most favoured when females with higher fitness can tolerate a large number of encounters with nonpreferred males relative to females with lower fitness. When costs of mate choice are assumed, an internal equilibrium of both the character and the preference alleles can occur, although allele frequencies usually follow a complex path to equilibrium.

Relatively few models of sexual selection can explain how a female mating preference controlled by a single genetic locus can evolve from a low initial frequency to observed values: how does a preference enter the population? Under the Fisherian process alone, the genetic correlation between alleles controlling male character and its female preference is generally too low to cause the latter's frequency to rise far from its initial value before variation in the preferred character is exhausted (O'Donald, 1980). Fisher's process under cyclical natural selection (Tomlinson, 1988b) and the handicap principle (Pomiankowski, 1987a) are two models that can cause higher preference frequencies to evolve. Each has its effects in different ways: in the former model, variation in the male character is 
maintained allowing preference more time to spread; and in the latter, preference is correlated with increased heritable fitness and is 'pulled' further through the population as a result. Models of female choice which assume polygenic control of preference (e.g. Lande, 1981) can show preference spreading far through a population. Although these models are elegant and instructive, they do not usually consider the question of how preference initially evolves. Simulating the evolution of preferences and preferred characters under the handicap models suggests ways in which preference can evolve to frequencies as high as those observed. Disadvantageous male characters can spread through the population to fixation or internal equilibria. The costs of female choice can also be overcome in some circumstances. Hence, the Fisherian process is reinforced. These results occur because, in comparison with Fisher's $(1930,1958)$ model, preferred males do not just gain more mates: they also gain fitter mates and hence fitter offspring. Females mating with preferred males also increase the chances of their offspring gaining extra fitness from their 'motherin-law'.

It is not necessary to invoke unrealistic coefficients of selection for the handicap models to have significant effects on the Fisherian process. The main purpose of the above models is not, however, quantitative, nor is it to study in detail the rival models of how female fitness interacts with preference expression. For example, the models necessarily make unrealistic assumptions about the genetic control of and variation in heritable fitness. What we can conclude qualitatively is that the Fisherian process can be influenced significantly and positively, not only by differences in male fitness, but also by differences in female fitness. Such mechanisms may apply not just to special cases of sexual selection by female choice, but may be of widespread importance. The models also show that populations in which a female preference is evolving may follow complex, nonrapid and nonintuitive paths to equilibrium. Unless the population is sampled at equilibrium, or followed over many generations, it will be difficult to draw conclusions about the role of sexual selection.

Theoretical evidence is accumulating that the evolution of female preferences and male characters depends greatly on the particular characteristics of a population. Fisher's process is probably correct as a basic model underlying the coevolution of preferences and characters. However, it is profoundly influenced by many factors: the mating system (O'Donald, 1983); the genetic control of traits
(O’Donald, 1980; Lande, 1981; Kirkpatrick, 1982); the existence of heritable variation in fitness (Zahavi, 1975; Andersson, 1986; Pomiankowski, 1987a; Tomlinson, 1988a); directional bias in mutations (Pomiankowski et al., 1991); spatial and temporal variation in natural selection acting on the preferred character (Lande, 1982; Charlesworth, 1988; Tomlinson, 1988b,c); the costs of choice (Pomiankowski, 1987b; Iwasa et al., 1991; Pomiankowski et al., 1991); the coexistence of more than one preferred character and preference (Tomlinson \& O’Donald, 1989; Pomiankowski \& Iwasa, 1993); and chance effects such as genetic drift. Female choice may be expected to vary widely and in an unpredictable way between species. Given the complex interactions of natural and sexual selection that determine the evolutionary outcomes, useful generalizations are difficult to make. Some species have clearly been the object of strong sexual selection (for example, the ruff or some species of widow birds); other species in the same family show no sexual dimorphism.

\section{References}

ANDERSSON, M. 1986. Evolution of condition-dependent sex ornaments and mating preferences: sexual selection based on viability differences. Evolution, 40, 804-816.

CHARLESWORTH, B. 1988. The evolution of mate choice in a fluctuating environment. J. Theor. Biol., 130, 191-204.

FISHER, R. A. 1930. The Genetical Theory of Natural Selection. Clarendon Press, Oxford.

FISHER, R. A. 1958. The Genetical Theory of Natural Selection, 2nd edn. Dover Publications, New York.

HAMILTON, w. D. AND ZUK, M. 1982. Heritable true fitness and bright birds: a role for parasites? Science, 218, 384-387.

IWASA, Y., POMIANKOWSKI, A. AND NEE, S. 1991. The evolution of costly mate prefernces. II. The 'handicap' principle. Evolution, 45, 1431-1442.

JOHN, J. L. 1995. Haematozoan parasites, mating systems and colorful plumages in songbirds. Oikos, 72, 395-401.

KIRKPATRICK, M. 1982. Sexual selection and the evolution of female choice. Evolution, 36, 1-12.

LANDE, R. 1981. Models of speciation by sexual selection on polygenic traits. Proc. Natl. Acad. Sci. U.S.A., 78, 3721-3725.

LANDE, R. 1982. Rapid origin of sexual isolation and character divergence in a cline. Evolution, 36, 213-223.

o'donald, P. 1980. Genetic Models of Sexual Selection. Cambridge University Press, Cambridge.

o'donald, P. 1983. The Arctic Skua. Cambridge University Press, Cambridge.

O'DONALD, P. AND MAJERUS, M. E. N. 1992. Non-random mating in the two-spot ladybird, Adalia bipunctata. III. New evidence of genetic preference. Heredity, 69 , 
$521-526$.

POMIANKOWSKI, A. 1987a. Sexual selection: the handicap principle. Proc. R. Soc. B, 231, 123-145.

POMIANKOWSKI, A. 1987b. The costs of choice in sexual selection. J. Theor. Biol., 128, 195-218.

POMIANKOWSKI, A. AND IWASA, Y. 1993. Evolution of multiple sexual preferences by Fisher's runaway process of sexual selection. Proc. R. Soc. B, 253, 173-181.

POMIANKOWSKI, A., IWASA, Y. AND NEE, s. 1991. The evolution of costly mate preferences. I. Fisher and the biased mutation. Evolution, 45, 1422-1430.

READ, A. F. 1987. Comparative evidence supports the Hamilton and Zuk hypothesis on parasites and sexual selection. Nature, 328, 68-70.

READ, A. F. AND HARVEY, P. H. 1989. Reassessment of comparative evidence for Hamilton and Zuk theory on the evolution of secondary sexual characters. Nature, 339, 618-620.

TOMLinson, I. P. M. 1988a. Diploid models of the handicap principle. Heredity, 60, 283-293.

TOMLINSON, I. P. M. 1988b. Major-gene models of sexual selection under cyclical natural selection. Evolution, 42, 814-816.

TOMLINSON, 1. P. M. 1988c. Models of clines in sexual selection by females choice. Biol. J. Linn. Soc., 36, 331-348.

TOMLINSON, 1. P. M. AND O'DONALD, P. 1989. The co-evolution of multiple male characters and female mating preferences: the 'gene-for-gene' model of sexual selection. J. Theor. Biol., 139, 219-238.

West-eberhard, M. J. 1979. Sexual selection, social competition and evolution. Proc. Am. Phil. Soc., 123, 222-234.

YEZERINAC, S. M. AND WEATHERHEAD, P. J. 1995. Plumage coloration, differential attraction of vectors and hematozoa infections in birds. J. Anim. Ecol., 64, 528-537.

ZAHAV1, A. 1975. Mate selection - a selection for a handicap. J. Theor. Biol., 53, 205-214.

\section{Appendix: details of the model}

The population is diploid, infinite and mates polygynously. In all the models, $A$ is the preferred character allele at locus $A, B$ is the preference allele at locus $\mathrm{B}$, and $C$ is the extra fitness allele at locus C. All loci are unlinked and alleles $A, B$ and $C$ are completely dominant over their alleles $a, b$ and $c$.

In general, we denote the frequencies of alleles by $\mathrm{p} A, \mathrm{p} B$, etc., the frequencies of adult genotypes by $\mathrm{p} A a B b C c$ etc., the frequencies of gametic genotypes by $\mathrm{p} A b C$ etc. and the frequencies of phenotypes by pAbC etc.

Before simulation commences, initial allele frequencies at each locus are specified and combined to produce adult genotypes in HardyWeinberg proportions. At any time, when new fitness alleles arise after the frequency of the old fitness allele has risen above 0.995 , the old fitness allele is assumed to have become fixed and no longer to be associated with extra fitness. The new fitness allele then becomes $C$ and is introduced at the specified frequency (see text and Tables 1 and 2) into all genotypes in linkage equilibrium with alleles at the $\mathrm{A}$ and $\mathrm{B}$ loci.

Simulations commence and typically are continued for several thousand generations. Matings occur initially. They are best analysed by subdivision into preferential and nonpreferential (random) matings, because females mate only once, whereas males can mate any number of times.

Consider first the matings of females expressing a mating preference for males of phenotype $\mathbf{A}$. If the proportion of females that mate preferentially is $M$, then in the $\mathrm{CE}$ and $\mathrm{CC}$ models,

$$
\begin{aligned}
M= & \mathrm{p} \mathbf{B C} \\
= & \mathrm{p} A A B B C C+\mathrm{p} A A B B C c+\mathrm{p} A A B b C c+\mathrm{p} A A B b C c \\
& +\mathrm{p} A a B B C C+\mathrm{p} A a B B C c+\mathrm{p} A a B b C c+\mathrm{p} A a B b C c \\
& +\mathrm{p} a a B B C C+\mathrm{p} a a B B C c+\mathrm{p} a a B b C c+\mathrm{p} a a B b C c
\end{aligned}
$$

and in the ED model,

$$
\begin{aligned}
M= & \mathrm{p} \mathbf{B C} *\left[1-(1-\mathrm{pA})^{n_{1}}\right]+\mathrm{pBC} *\left[1-(1-\mathrm{pA})^{n_{2}}\right] \\
= & {\left[1-(1-\mathrm{pA})^{n_{1}}\right] *[\mathrm{p} A A B B C C+\mathrm{p} A A B B C c} \\
& +\mathrm{p} A A B b C c+\mathrm{p} A A B b C c+\mathrm{p} A a B B C C+\mathrm{p} A a B B C c \\
& +\mathrm{p} A a B b C c+\mathrm{p} A a B b C c+\mathrm{p} a a B B C C+\mathrm{p} a a B B C c \\
& +\mathrm{p} a a B b C c+\mathrm{p} a a B b C c] \\
& +\left[1-(1-\mathrm{pA})^{n_{2}}\right] *[\mathrm{p} A A B B c c+\mathrm{p} A A B b c c \\
& +\mathrm{p} A a B B c c+\mathrm{p} A a B b c c+\mathrm{p} a a B B c c+\mathrm{p} a a B b c] .
\end{aligned}
$$

Female gamete genotype frequencies $(\mathrm{p} A B C$, $\mathrm{p} A B C$, etc.) for preferential matings are calculated from the frequencies of the adult genotypes of those females expressing a preference. Male gamete genotype frequencies are calculated from the adult genotype frequencies among preferred males (frequency pA) only. Because the total frequency of preferential matings must be $M$, male gamete genotype frequencies are normalized to unity by division of each by pA. Male and female gametes in preferential matings are then combined according to a Punnett square to give the frequencies of the genotypes of the offspring of these matings. Hence,

$\mathrm{p} A A B B C C=\mathrm{p} A B C * \mathrm{p} A B C$ etc.

For random matings, frequency $1-M$, the gamete frequencies from females not expressing a preference are determined in the same fashion as those for females expressing a preference. Because all males can take part in random matings, male gamete frequencies are calculated from the whole population of adult male genotypes (which, of course, already sum to one). Male and female gametes in 
random matings are then combined according to a Punnett square to give the frequencies of the genotypes of the offspring of these matings.

Natural selection then acts if choice is costly. The frequency of individuals derived from preferential matings is reduced by $\left(1-S_{\text {cost }}^{\prime}\right)$ where $S_{\text {cost }}^{\prime}=$ $S_{\text {cost }}^{\prime} * M / \mathrm{pA}$ and $S_{\text {cost }}$ is of some specified value (zero in the CE and ED models). The frequency of individuals in the next generation is then normalized to unity using the denominator $(1-M)+\left(M * S_{\text {cost }}^{\prime}\right)$.

Natural selection then acts additively on the male character genotypes $A A$ and $A a$ and the extra fitness genotypes $C C$ and $C c$. pAc is reduced by $\left(1-S_{\mathrm{a}}\right)$ and paC is increased by $\left(1+S_{\mathrm{c}}\right)$, where $S_{\mathrm{a}}$ and $S_{\mathrm{c}}$ are specified. pAC is increased/reduced by $\left(1-S_{\mathrm{a}}+S_{\mathrm{c}}\right)$. The frequency of individuals is then normalized to unity using the denominator

$$
1-\left[(\mathrm{pAc}+\mathrm{pAC}) * S_{\mathrm{a}}\right]+\left[(\mathrm{paC}+\mathrm{pAC}) * S_{\mathrm{c}}\right] \text {. }
$$

It is convenient to output allele ( $\mathrm{p} A, \mathrm{p} a$, etc.), genotype $(\mathrm{p} A a B b C c$, etc.) and phenotype ( $\mathrm{pA}, \mathrm{pa}$, etc.) frequencies and to calculate correlation coefficients at this point. The cycle then repeats for the next generation and new mating frequencies are calculated. Equilibrium is reached when no allele frequency changes by more than $10^{-6}$ over 100 generations. 